\title{
R-spondin-mediated WNT signaling potentiation in mammary and breast cancer development
}

\author{
Johanna M. Tocci ${ }^{1,2}$ ｜ Carla M. Felcher ${ }^{1,2}$ ｜ Martín E. García Solá ${ }^{1,2}$ | \\ Edith C. Kordon ${ }^{1,2}$ (1)
}

${ }^{1}$ Instituto de Fisiología, Biología

Molecular y Neurociencias (IFIBYNE),

CONICET-Universidad de Buenos Aires,

Buenos Aires, Argentina

${ }^{2}$ Departamento de Química Biológica, Facultad de Ciencias Exactas y Naturales, Universidad de Buenos Aires, Buenos

Aires, Argentina

Correspondence

Edith C. Kordon, IFIBYNE-UBA-

CONICET, Ciudad Universitaria, Ciudad

Autónoma de Buenos Aires 1428,

Argentina.

Email: ekordon@qb.fcen.uba.ar

\section{Funding information}

Consejo Nacional de Investigaciones

Científicas y Técnicas; Fondo para la

Investigación Científica y Tecnológica,

Grant/Award Number: PICT2016-2834;

Universidad de Buenos Aires, Grant/

Award Number: UBACyT

20020160100087BA

\begin{abstract}
The mammary gland is a secretory organ, which develops as a network of growing epithelial ducts composed of luminal and basal cells that invade the surrounding adipose tissue through a series of developmental cycles. Mammary stem cells (MaSCs) maintain an accurate tissue homeostasis, and their proliferation and cell fate determination are regulated by multiple hormones and local factors. The WNT pathway plays a critical role in controlling the enormous tissue expansion and remodeling during mammary gland development through the maintenance and differentiation of MaSCs, and its deregulation has been implicated in breast cancer $(\mathrm{BC})$ initiation and progression. The R-spondins (RSPOs) are four secreted proteins that strongly enhance target cell sensitivity to WNT ligands. Moreover, leucine-rich repeat-containing G-protein-coupled receptors (LGRs) 4-6 are considered obligate high-affinity receptors for RSPOs and have been described as stem cell markers. Importantly, elevated RSPO expression has been recently identified in several tumor types from patients, including $\mathrm{BC}$, and it has been reported that they play a significant role in mammary tumor progression in experimental models. In this review, exploring our present knowledge, we summarize the role of the RSPOLGR axis as a WNT-enhancing signaling cascade in the MaSC compartment and during the normal and neoplastic mammary gland development. In addition, we include an updated expression profile of the RSPOs and their action mediators at the cell membrane, the LGRs, and the ubiquitin-ligases ZNRF3/ RNF43, in different BC subtypes. Finally and based on these data, we discuss the significance of tumor-associated alterations of these proteins and their potential use as molecular targets for detection and treatment of BC.

K E Y W O R D S

breast cancer, LGR, mammary gland, RSPO, WNT
\end{abstract}

\footnotetext{
Abbreviations: BC, breast cancer; BCSCs, breast cancer stem cells; BR, basic amino acid-rich; EMT, epithelial-to-mesenchymal transition; HSPGs, heparan sulfate proteoglycans; MaSCs, mammary stem cells; TEBs, terminal end buds; TSR, thrombospondin type I repeat.
}

Johanna M. Tocci and Carla M. Felcher contributed equally to this study.

\section{1 | WNT PATHWAY OVERVIEW}

WNTs represent a numerous family of secreted lipidmodified, cysteine-rich glycoproteins which share a high degree of sequence homology. Activation of WNT 
signaling pathways has been described over the past decades as cell-biological programs critically required for several processes including embryonic development, regeneration, and cellular differentiation. Among pathological conditions, these pathways play a clear role in all steps of carcinogenesis, from tumor initiation to cancer growth and dissemination (all this information is wellreviewed in References 1 and 2). Since WNTs are hydrophobic as a result of posttranslational lipid modifications, ${ }^{3}$ they are unlikely to diffuse freely. Thus, once in the extracellular matrix (ECM), WNTs are most commonly restricted to autocrine and juxtacrine signaling. However, with the assistance of carrier structures like WNT-binding chaperones, heparan sulfate proteoglycans (HSPGs), lipoproteins, exosomes, and cytonemes, and even in a cell-bound manner through cell divisions, it has been suggested that WNTs can act as short- and relative long-range paracrine signaling molecules influencing the behavior of target cells in a concentration gradient-dependent manner. ${ }^{4-7}$ Even though there have been several reports trying to elucidate this WNT extracellular transport (well-reviewed in References 8 and 9), how exactly WNT gradients are formed and how shortand relative long-range WNT spreading is achieved in tissue homeostasis and development is still poorly understood.

At the cell membrane, WNT ligands mostly initiate signal transduction through binding to a member of the frizzled (FZD) transmembrane receptor family, but atypical receptors have been described, including the receptor tyrosine kinase (RYK)-like orphan receptor 1 or 2 (ROR1/2) and the RYK. ${ }^{10}$ The large family of WNT factors has long been grouped into two main categories, "canonical" and "noncanonical" ligands, as the downstream signaling pathways they activate. However, the intracellular response to each individual WNT is markedly context-specific, since several reports described the ability of distinct WNTs to activate both canonical and noncanonical WNT signaling pathways. ${ }^{10}$ Thus, the intracellular output might be the result of a combination between WNTs and a given receptor/co-receptor at the cell membrane, rather than the nature of each individual WNT. Since these cascades have been extensively explained in numerous reviews, ${ }^{1,11}$ they will only be described briefly herein.

Canonical WNT pathway, often referred to as $\mathrm{WNT} / \beta$-catenin signaling, is essential to several developmental processes by regulation of cell proliferation, differentiation, and migration, while its deregulation underlies a wide range of pathologies including cancer. After binding to FZD and a member of the low density lipoprotein-related protein 5 or 6 (LRP5/6) family, WNT ligands are able to activate the canonical WNT signaling pathway by inducing the release of $\beta$-catenin from a cytosolic, multiprotein destruction complex (Figure 1, upper panel). Therefore, $\beta$-catenin accumulates and translocates into the nucleus where it acts as a co-activator of the Tcell factor/lymphoid enhancer factor (TCF/LEF) family of transcription factors, promoting the transcription of a large number of target genes (reviewed in Reference 1).

Several noncanonical WNT pathways, that do not involve $\beta$-catenin, have been described (Figure 1, lower panel): as the $\mathrm{WNT} /$ planar cell polarity (PCP) pathway, in which FZD-bound WNTs establish a PCP and regulate polarized cell migration through modulation of the cytoskeletal factors, RhoA and JUN kinase (JNK); the WNT/Ca ${ }^{2+}$ pathway in which WNTs, through FZD binding, can trigger $\mathrm{Ca}^{2+}$ fluxes; the WNT-ROR2 binding that triggers an intracellular cascade to inhibit $\mathrm{WNT} / \beta$ catenin pathway by JNK activation; and the WNT-RYK binding that activate Src proteins (all four reviewed in Reference 10).

\section{1 | WNT pathway potentiators: The R- spondins}

R-spondins (roof plate-specific spondins; RSPOs) are a family of four secreted glycoproteins (RSPO1-4) that have highly conserved domain structures with two amino-terminal cysteine-rich furin-like repeats (FU1 and FU2) and a thrombospondin type I repeat (TSR) domain toward the basic amino acid-rich (BR) carboxyl terminal domain $\left.{ }^{12,13}\right)$. RSPOs markedly differ in the length of the BR carboxyl domain, being RSPO3 the largest, followed by RSPO $1 .{ }^{14}$ Once secreted, the RSPOs can bind to the leucine-rich repeat-containing G-protein-coupled receptors 4 , 5, or 6 (LGR4-6) ${ }^{15}$ through their FU2 domain and/or to the extracellular domains of two membranebound E3 ubiquitin ligases, Zinc and RING finger 3/RING finger 43 (ZNRF3/RNF43), through the FU1 domain. ${ }^{16}$ Once secreted, in culture, RSPOs have been either poorly detected or nearly undetected in the conditioned medium of cells that express them. ${ }^{12,17}$ As it was observed that adding soluble heparin or sodium chlorate, an inhibitor for sulfation, strongly enhanced free RSPO levels, it was concluded that these proteins remain bound to the cell surface and/or ECM components, like HSPGs. ${ }^{17}$ Moreover, this study demonstrated that deletions of the BR or both the TSR and BR domains turned the RSPOs easily detected as soluble proteins. ${ }^{17}$ More precisely, it has been shown that positively charged amino acids enriched in the BR domain, as well as the TSR domain itself, promoted RSPO cell surface retention by interacting with different types of Syndecan, ${ }^{18,19}$ one of the major membrane-bound HSPGs (see Figure 2). 

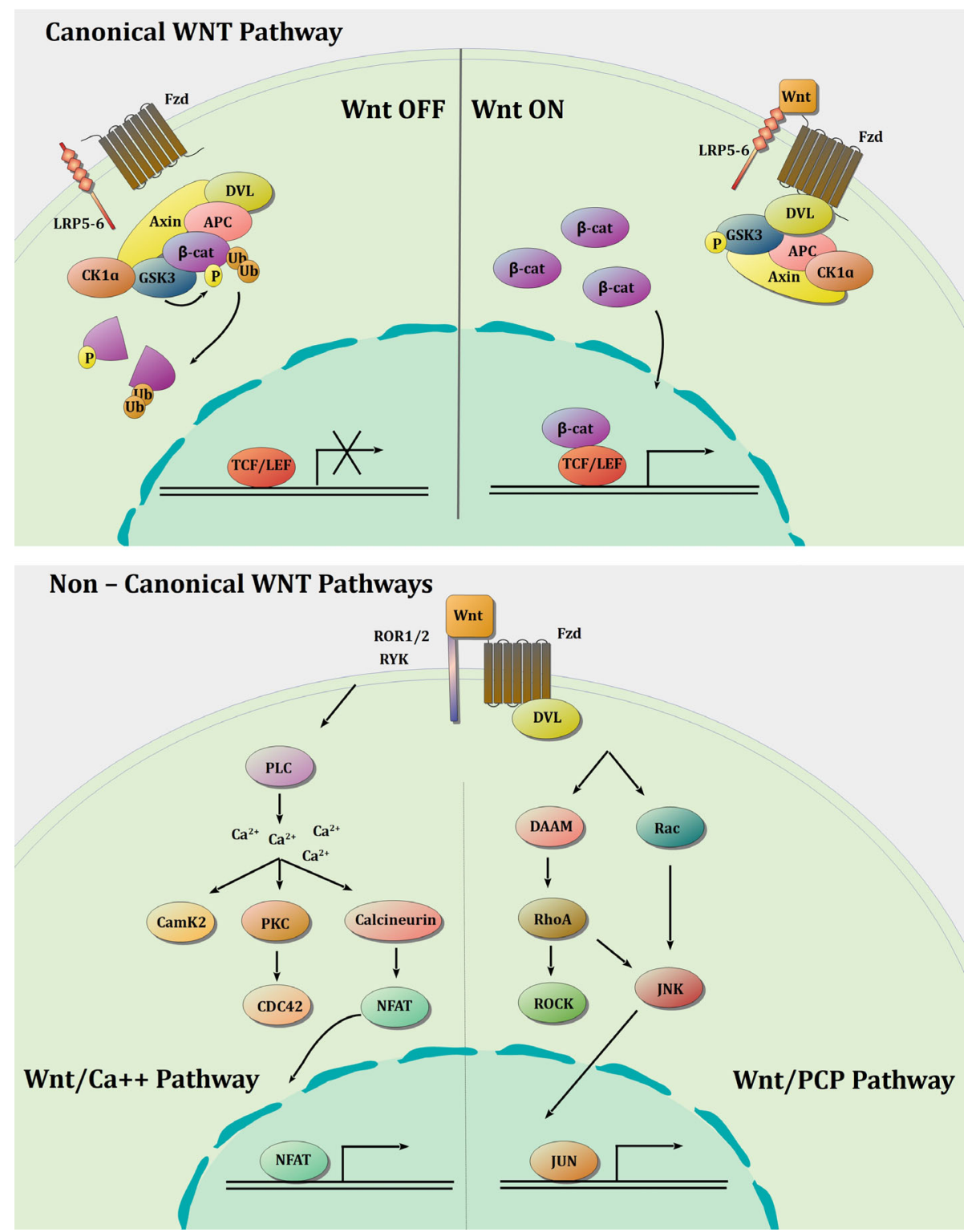

FI G U RE 1 Canonical WNT pathway (upper panel). WNT OFF. Without WNT ligands, cytosolic $\beta$-catenin is retained in a multiprotein destruction complex composed by Axin, APC, GSK-3, and CK1 $\alpha$, which leads to $\beta$-catenin phosphorylation, ubiquitination, and consequent degradation via proteasome. In the nucleus, TCF/LEF is in an inactive state and, therefore, the transcription of target genes is OFF (left). WNT ON. After WNT binds to its receptor FZD, and co-receptor LRP5/6, $\beta$-catenin is released from the destruction complex, since FZDbound DVL recruits the multiprotein complex contributing to its destabilization. This prevents phosphorylation and degradation of $\beta$-catenin, resulting in its accumulation and translocation into the nucleus where it acts as a co-activator of TCF/LEF, promoting the transcription of target genes (right). Noncanonical WNT pathways (lower panel). FZD-bound WNTs act in combination with different coreceptors activating $\beta$-catenin-independent mechanisms. The WNT/Ca2+ pathway (left) promotes the activation of phospholipase $\mathrm{C}$ (PLC) activity, leading to intracellular $\mathrm{Ca} 2+$ fluxes and downstream modulations that promote cytoskeletal rearrangements and/or transcriptional responses, as the NFAT nuclear activity. In the WNT/PCP pathway (right), DVL binds to Rho by de-inhibition of DAAM1. Rac1 and Rho together trigger ROCK and JNK activation. This leads to JUN transcription factor activation and/or rearrangements of the cytoskeleton. DVL, dishevelled; DAAM1, dishevelled-associatedactivator of morphogenesis 1; FZD, frizzled; JNK, JUN kinase; LRP5, lipoprotein-related protein 5; NFAT, nuclear factor of activated T cell; ROCK, rho-associated protein kinase; TCF/LEF, T-cell factor/lymphoid enhancer factor

Therefore, it may be expected that RSPOs exert their activities in an autocrine or short-range paracrine manner. However, it remains to be elucidated whether other HSPGs or extracellular components, and even the markedly different BR length among RSPOs, are involved in determining their location and spreading in different mammalian tissues.

LGR proteins are evolutionarily-conserved seventransmembrane receptors that belong to the $\mathrm{G}$ proteincoupled receptor superfamily. ${ }^{20}$ They contain a large 


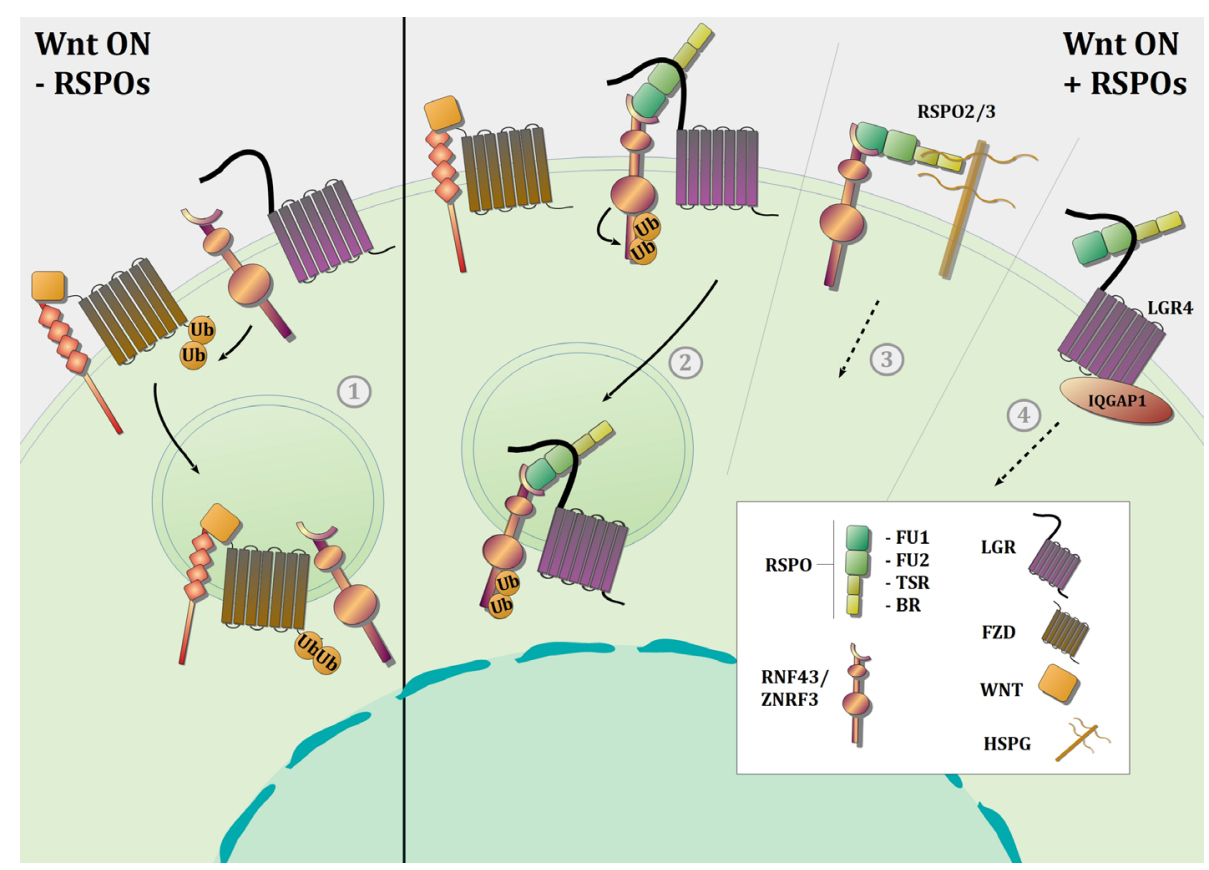

F I G U R E 2 WNT signaling amplification by RSPOs. Upon binding of WNT ligands to their receptors and co-receptors, target gene expression and/or rearrangements of the cytoskeleton are activated. Without RSPOs, a negative feedback mechanism maintains WNT signaling regulated due to the activity of transmembrane ubiquitin ligases, ZNRF3/RNF43, identified as negative modulators of the WNT signaling. These related ligases decrease the availability of FZD promoting membrane clearance through endocytosis and ubiquitin-mediated degradation. ${ }^{1}$ With RSPOs, WNT signaling is potentiated by three distinct mechanisms: RSPOs bind to LGR4/5/6 and ZNRF3/RNF43 triggering the auto-ubiquitination of ZNRF3/RNF43, increasing the availability of FZD receptors. ${ }^{2}$ RSPO2 and RSPO3 potentiate WNT signaling by interacting with ZNRF3/RNF43 and membrane-bound HSPGs, as Syndecans, in an LGR-independent manner. ${ }^{3}$ The RSPOLGR4 complex enhances WNT pathways through the recruitment of IQGAP1 in a ZNRF3/RNF43-independent mechanism. ${ }^{4}$ HSPG, heparan sulfate proteoglycan; IQGAP1, IQ motif containing GTPase-activating protein 1; LGR, leucine-rich repeat-containing G-protein-coupled receptor; RSPO, R-spondin; ZNRF3/RNF43, zinc and RING finger 3/RING finger 43

extracellular region (ectodomain) with multiple imperfect copies of a leucine-rich repeat protein interaction domain. ${ }^{20}$ Structural equivalences among LGR 4, 5, and 6 have been pointed due to sequence alignment of their ectodomains. ${ }^{21}$ LGR4-6 were considered orphan receptors until 2011, when it was demonstrated that the RSPOs were their high-affinity ligands. ${ }^{15}$

Transmembrane E3 ubiquitin ligases ZNRF3 and RNF43 negatively regulate the WNT pathways by promoting endocytosis and turn-over of FZD and LRP6 in the absence of RSPOs ${ }^{22,23}$ (Figure 2). However, RSPO-to-LGR binding induces auto-ubiquitination and membrane clearance of ZNRF3/RNF43, promoting FZD accumulation at the cell surface and enhancing the activation of canonical and noncanonical WNT signaling pathways ${ }^{22-24}$ (Figure 2).

Additional mechanisms that explain RSPO potentiating ability have been reported. Upon RSPO stimulation, LGR4/5 binds to LRP5/6 and FZD enhancing canonical WNT signaling. ${ }^{25,26}$ Besides, it has been demonstrated that on top of RNF43/ZNRF3 clearance, the RSPO-LGR4 complex is also able to potentiate WNT pathways through the recruitment of the intracellular scaffold IQ motif containing GTPase-activating protein 1 (IQGAP1), in a ZNRF3-independent manner. ${ }^{27}$ Moreover, three recent independent studies have reported that RSPO2 and RSPO3 are able to enhance WNT signaling by interacting with ZNRF3/RNF43, without the requirement of LGR binding, but involving the interaction with membrane-bound HSPGs by the TSR/BR domains ${ }^{28-30}$ (Figure 2). Considering these data and the fact that HSPGs are expressed ubiquitously on the surface of most cells $^{31}$ whereas LGRs are specifically expressed in some stem cell niches, ${ }^{20}$ it could be suggested that an RSPO-toLGR binding might be relevant when a high level of a WNT signaling pathway must be activated in a specific cell compartment, as in the stem cells of various tissues, while an HSPG-mediated RSPO signaling may allow a less specific WNT pathway activation during the development of different tissues. Therefore, given the fact that several RSPO receptors have been identified in various experimental models, further studies are required to clarify how RSPO proteins may utilize different receptors or a combination of different receptors depending on each specific context to amplify target cell responses to WNT ligands. 
Functionally, and based on their ability to potentiate WNT pathway activation, the RSPOs play critical roles in embryonic development and organogenesis as well as in the self-renewal and maintenance of stem cells in several tissues (reviewed in References 14 and 32). Particularly, they have been implicated in sex determination, vasculature formation, and in the development and growth of lungs, hairs, nails, limbs, intestine crypts, and muscles (reviewed in References 14 and 32). Coincidentally, the LGRs have been described as stem cell markers and WNT-regulated target genes in different tissues, playing a relevant role in embryonic development and adult tissue homeostasis. $^{20,33,34}$

Under pathological conditions, elevated RSPO expression due to genomic rearrangements or transcriptional activation has been identified in mouse and human tumors, ${ }^{35,36}$ suggesting that these proteins may be functionally relevant for tumor development. Acting through canonical or noncanonical WNT pathway activation, the RSPOs have been implicated in the progression of several cancer types including colorectal, ovarian, lung, bladder, and breast cancer (BC) ${ }^{36-38}$ Here, we focus on the role of RSPO-LGR signaling axis in the normal and neoplastic mammary gland development.

\section{2 | MAMMARY GLAND DEVELOPMENT AND BC: OVERVIEW}

The mammary gland is an epithelium formed by an inner luminal sheet surrounded by an outer layer of myoepithelial or basal cells. ${ }^{39}$ This tissue is an exocrine gland of ectodermal origin, whose development starts during embryogenesis and continues during puberty. Since the fundamental aspects of mammary tissue organization and development are well conserved between rodents and humans, mice and rats have been commonly used as experimental models to analyze developmental processes and oncogenic pathways. ${ }^{40}$ Mammary gland formation in mouse embryos starts on the ectoderm at mid gestation, around 10 days after mating has occurred (E10). In 2 days (by E12, approximately), five (three thoracic and two inguinal) mammary placodes develop into mammary buds at each flank. In males, the mesenchyme around these structures expresses the androgen receptor that leads to the destruction of the mammary anlage in many mouse strains. In female embryos, the buds remain quiescent until E15.5-E16.5, when mammary epithelial cells start to divide and the buds sprout into the developing mammary fat pads located within the dermis. Then, a process of ductal branching begins, giving rise to the rudimentary ductal tree observed at birth. ${ }^{40}$
Fetal human and mouse mammary development were reported to be quite parallel. ${ }^{41}$ Nevertheless, the human process is more complex involving 10 phases associated with increasing embryonic length. ${ }^{42}$ Besides, in the human fetal gland, most epithelial cells are positive for both luminal and basal markers (CK19 and CK14, respectively) and only near delivery basal and luminal signatures can be distinguished as either $\mathrm{C} 4^{+}$or $\mathrm{CK}_{1} 9^{+}$cells; however, only $\mathrm{CK} 14^{+}$cells have been observed in embryonic mouse mammary glands. Another difference at birth is that in female human newborns various minor ductal trees converge at each nipple, while in mice, a single ductal network corresponds to each gland. ${ }^{41}$

Before puberty, mammary epithelium grows according to the body size. At the beginning of puberty (approximately 5 weeks after birth in mice and 8-12 years of age in humans), the release of ovarian hormones promotes ductal development and branching of the gland. In addition, in both species, there is a renewed growth of mammary stroma (fatty and fibrous tissue) at this time. During this phase, at the distal ends of the mammary ducts, bulbous structures composed by multiple layers of cuboidal epithelial cells called terminal end buds (TEBs) can be observed. They constitute the invading fronts of the ducts, which extend and branch by bifurcation until reaching the limits of the fat pad at 8-12 weeks in mice and 18-24 years in humans whereupon the TEBs regress. ${ }^{41,43}$ Then, in the mature nulliparous female, ductal trees with budding structures (mouse alveolar buds or human terminal duct lobular units, TDLUs), each of which correspond to a group of acini from a single terminal bud, can be observed. One significant difference between mammary glands of puberal humans and mice is that in the first, lobules develop at this stage, while in the second these structures only appear if pregnancy occurs. In both species there is an extensive ductal branch expansion from the mouse alveolar buds or human TDLUs at the initiation of pregnancy. Finally, just before delivery, mammary differentiation reaches its maximum with the development of alveoli that produce lipids and protein for postpartum milk secretion. ${ }^{41}$

Twenty years ago, using serial transplantation of retrovirally tagged mammary cells, it was demonstrated that certain individual cells were able to completely reconstitute normal mammary tissue upon transplantation into de-epithelialized (cleared) mammary fat pads of host syngeneic mice. ${ }^{44}$ This study provided the basis of our current understanding of the mammary gland cellular hierarchy, based on the existence of multipotent mammary stem cells (MaSCs) that can reconstitute the whole gland upon transplantation into cleared mammary fat 
pads. ${ }^{45}$ However, lineage-tracing studies demonstrated that the mammary gland embryonically develops from bipotent MaSCs that are later replaced by unipotent progenitors, which show their enormous potential during puberty and in the successive pregnancies. ${ }^{39}$ These unipotent progenitors produce two functionaldifferentiated subtypes: the hormone-sensing or secretory luminal cells, and the basal myoepithelial contractile cells. ${ }^{46}$ Interestingly, the multipotent stem cell activity of these progenitors is revealed in the transplantation assays. Physiologically, this capacity may be very important for recovery from local lesions or diseases, such as mastitis. ${ }^{46}$ In addition, it has also been shown that localized stem cells originate clonal regions within the epithelial tree and that there are progenitor cell populations restricted to lobular or ductal development (reviewed in Reference 46).

BC-initiating cells or BC stem cells (BCSCs) are subpopulations of breast tumor cells enriched for clonogenic activity, which play critical roles in tumor initiation, progression, therapy resistance, and cancer recurrence. ${ }^{47} \mathrm{It}$ has been determined that human $\mathrm{BC}$ cells that were positive for the antigen CD44 and had no or low expression of CD24 were enriched for tumorigenic activity in xenografts grown in immuno-compromised mice. ${ }^{47}$ Therefore, $\mathrm{CD} 44^{+} / \mathrm{CD} 24^{\mathrm{lo} /-}$ have been considered as a BCSCenriched population. MaSCs and BCSCs share some fundamental features such as self-renewal capability and multilineage potency. However, the first are not necessarily the origin of the second, since more differentiated cells can acquire mutations resulting in dedifferentiation, gain of self-renewal capabilities and epithelial-tomesenchymal transition (EMT) (reviewed in Reference 46). In spite of that, it is often proposed that tumors arise from transformed MaSCs that acquire heterogeneity through clonal evolution, especially because they are long-lived and therefore more likely to accumulate mutations (reviewed in Reference 46). The concept that a mammary neoplasia is commonly originated from MaSCs suggests that determinant factors for cell fate decisions during normal development, such as WNT pathway regulators, are likely to be crucial in defining tumor phenotype and therapy response.

Five intrinsic molecular subtypes of $\mathrm{BC}$ have been described. ${ }^{48}$ They are known as Luminal A, Luminal B, HER2-enriched, Basal-like, and Claudin-low. When analyzed in combination with clinical data (tumor grade, hormone receptor status, and HER2 status), they display relevant differences in their incidence, risk factors, prognosis, and treatment sensitivity. ${ }^{48}$ Luminal tumor cells resemble those lining the normal mammary ducts. Most Luminal A and B tumors express estrogen receptors $\left(\mathrm{ER}^{+}\right)$. However, Luminal A, the most common subtype (30-70\% of BCs), tend to display a negative HER2 status
$\left(\mathrm{HER}^{-}{ }^{-}\right.$), that is, they do not show amplification/overexpression of the HER2 gene, whereas Luminal B can be either HER2 positive or negative. Luminal BCs normally respond to hormone therapy and their prognosis has a favorable outlook, particularly the Luminal A subtype, when treated early. ${ }^{48}$ The HER2-enriched subtype represents $5-15 \%$ of BCs. Although not all tumors expressing HER2 belong to this group, most of them are HER2 ${ }^{+}$as well as estrogen and progesterone receptor negative $\left(\mathrm{ER}^{-} \mathrm{PR}^{-}\right)$. Tumors within this subtype tend to grow fast and be invasive; however, specific anti-HER2 therapeutic approaches have improved their treatment. ${ }^{48}$ The Basallike category is the most distinct among all the molecular subtypes and it is defined by a robust cluster of genes expressed by epithelial cells in the basal or outer layer of the adult mammary gland. Most of the Basal-like tumors are triple-negative $\left(\mathrm{ER}^{-} \mathrm{PR}^{-} \mathrm{HER} 2^{-}\right)$and have a highly aggressive behavior. Thus, they have less specific therapeutic options and the worst prognosis among all BC subtypes. ${ }^{49}$ Finally, the Claudin-low tumors, which have been more recently characterized, show low to none expression of luminal markers, high enrichment for EMT markers, and cancer stem cell-like features. Therefore, it has been hypothesized that the most primitive MaSCs may be the cellular origin for this subtype. ${ }^{50}$ Clinically, most Claudin-low tumors are triple-negative with a poor prognosis and a high frequency of metaplastic differentiation, and their therapeutic options are similar to those available for the Basal-like subtype. However, it has been reported that the Claudin-low signature is enriched in residual tumor tissue remaining after either endocrine therapy or chemotherapy, ${ }^{50}$ indicating that finding specific targets for its treatment would have an important impact in preventing $\mathrm{BC}$ relapse.

\section{3 | WNT PATHWAY INVOLVEMENT IN MAMMARY GLAND DEVELOPMENT AND NEOPLASIA}

WNT signaling has been implicated in determining the specification and morphogenesis of mammary cell populations during almost all stages of embryonic and postnatal mammary gland development (well-reviewed in References 51 and 52).

\section{1 | Canonical WNT pathway in the mammary gland}

During mouse embryogenesis, the canonical WNT pathway determines the cell fate of those composing the 
mammary lines and placodes. ${ }^{53}$ Inhibition of distinct WNT-related factors resulted in placodal impairments, while hyperactivation of $\mathrm{WNT} / \beta$-catenin signaling led to their accelerated induction. ${ }^{54,55}$ During postnatal mammary development, WNT/ $\beta$-catenin signaling plays a critical role in controlling the enormous tissue expansion and remodeling (i.e., branching morphogenesis, alveolar bud formation, and early lobulo-alveolar development during pregnancy) through the maintenance and differentiation of MaSCs within the basal mammary epithelium. ${ }^{55,56}$

Several studies have demonstrated mammary tumor development upon canonical WNT pathway hyperactivation using different mouse models. Since WNT/ $\beta$ catenin activation regulates MaSC dynamics, it is expected that its pro-oncogenic capacity would reside in a deregulated, altered stem/progenitor cell expansion. In fact, overexpression of WNT1 or activated $\Delta$ N89 $\beta$-catenin led to mammary hyperplasias that displayed stem/progenitor markers in vivo and enhanced colony-forming ability and bipotency in culture. ${ }^{54,57}$ Moreover, canonical WNT signaling hyperactivation in BC was involved in BCSC survival, bulk-tumor expansion, and enhanced invasion. High cytoplasmic and nuclear accumulation of $\beta$-catenin has been detected in approximately $60 \%$ of human BCs, ${ }^{58}$ particularly in the Basal subtype ${ }^{59,60}$ associated with an increase in the $\mathrm{CD}_{4}{ }^{+} \mathrm{CD}^{2} 4^{-}$stem-cell-like population. ${ }^{59} \mathrm{WNT} / \beta-$ catenin activation was also observed in mouse-derived basal-like tumors that exhibited characteristics of this human BC subtype. ${ }^{61}$ Likewise, canonical WNT signaling blockage decreased the migratory potential and growth of Basal-like BC cells in culture and in several mouse xenograft models of triple-negative $\mathrm{BC} .^{62,63}$ However, canonical WNT signaling activation in $\mathrm{BC}$ is not commonly induced by $\beta$-catenin mutations. Therefore, studying new factors involved in its modulation may lead to new therapeutic strategies to control BC progression.

\section{2 | Noncanonical WNT pathway in the mammary gland}

Although WNT/ $\beta$-catenin signaling has been extensively studied and is clearly implicated in mammary gland development, it is known that noncanonical WNT pathways also modulate mammary stem/progenitor biology. WNT5A and WNT5B represent two noncanonical WNT ligands that play a role within the mammary epithelium, and whose expression is restricted to mature luminal epithelial cells. ${ }^{64}$ Particularly, WNT5A inhibited branching morphogenesis ${ }^{65}$ as a result of WNT5A/ ROR2 interaction at the cell membrane ${ }^{66}$ However, the same WNT ligand was able to promote mammary epithelial progenitor growth through RYK interaction, ${ }^{66}$ suggesting that WNT5A may increase MaSC capacity and proliferation or inhibit mammary morphogenesis in a noncanonical RYK-specific manner. In addition, WNT5B has been described as a negative regulator of mammary stem/progenitor function by inhibition of the WNT/ $\beta$-catenin signaling and activation of the noncanonical $\mathrm{Ca}^{2+} /$ nuclear factor of activated $\mathrm{T}$ cell (NFAT) cascade. ${ }^{67}$ Besides, overexpression of WNT5B resulted in strongly decreased mammary branching morphogenesis and impaired epithelial outgrowth. ${ }^{67}$

WNT/ $\beta$-catenin signaling has also been more frequently implicated in mammary tumorigenesis. However, some studies have shown that the noncanonical WNT cascades have relevant implications on BC clinical outcome. $^{68}$ In mice, WNT5a-knockdown inhibited both canonical and noncanonical WNT pathways, leading to suppressed invasion and sphere formation of basal-like mammary tumor cells. ${ }^{69}$ In addition, it was determined that the alternative WNT receptor ROR2 is expressed in different BC subtypes, including Luminal, Basal-like, and Claudin-low, whereas canonical WNT signaling is preferentially restricted to the basal-like subtype. ${ }^{61}$ Interestingly, ROR2 expression was negatively correlated with $\mathrm{WNT} / \beta$-catenin-dependent pathway activity in mouse tumors as well as in human breast tumor samples, establishing an inverse correlation between $\mathrm{WNT} / \beta$-catenindependent and WNT/ $\beta$-catenin-independent pathways. ${ }^{61}$ In the TP53-null model, Ror 2 depletion inhibited tumor growth and cell adhesion, increasing WNT/ $\beta$-catenin activation and cell migration. ${ }^{61}$ In human basal-like BC cells, silencing ROR1—also involved in noncanonical WNT pathway activation-impaired EMT, cell migration, invasion, and metastatic potential. ${ }^{70}$ Besides, high ROR1 expression was tightly correlated with poor metastasisfree survival of BC patients. ${ }^{70}$

Taken together, these studies show the complexity of the WNT pathway activity in the mammary gland. However, a deeper understanding of the specific WNT signaling function depending on the existing repertoire of WNT ligands, receptors, modulators, and downstream signaling components in normal human breast development and among the different BC subtypes is still missing. Moreover, deciphering the specific molecular mechanisms altered as a result of a WNT signaling deregulation will also provide valuable information to select the patient subsets that may benefit from the development of therapeutic drugs targeting this pathway. 


\section{I RSPO/LGR AXIS IN MAMMARY GLAND AND BC}

\section{1 | RSPOs in normal mammary gland development}

It is well-documented that the RSPOs play critical roles in embryonic development and organogenesis as well as in the self-renewal and maintenance of stem cells in several adult tissues. ${ }^{14,32}$ Regarding the mammary gland, it was reported that $\operatorname{RspoI}^{(-/-)}$mice were unable to feed their pups, ${ }^{71}$ and that transplants of their glands into cleared fat pads of wild-type mice showed impaired sidebranching in virgin females and reduced alveolar formation with a persistence of virgin markers during pregnancy. ${ }^{72,73}$ Importantly, it has been shown that RSPO1 expression is induced in $\mathrm{ER}^{-} \mathrm{PR}^{-}$mammary cells of wildtype mice during pregnancy or upon treatment with estrogen, progesterone, or both hormones. ${ }^{74}$ These results indicated that sex hormones would cause the secretion of a mediator that, in turn, would trigger RSPO1 synthesis in $\mathrm{ER}^{-} \mathrm{PR}^{-}$mammary cells. In addition, it was shown that the maintenance of the MaSC compartment in vivo requires the presence of both RSPO1 and WNT4, which is expressed by $\mathrm{ER}^{+} \mathrm{PR}^{+}$cells, to fully activate the $\mathrm{WNT} / \beta$-catenin signaling in the progenitor population. ${ }^{74}$ Then, it was demonstrated that progesterone indirectly stimulates RSPO1 expression in the mammary gland through induction of the receptor activator of nuclear factor kappa $\mathrm{B}$ ligand in $\mathrm{PR}^{+}$cells. ${ }^{75}$ More recently, amphiregulin was identified as a relevant factor, secreted by $\mathrm{ER}^{+}$cells, also able to upregulate RSPO1 in luminal $\mathrm{ER}^{-}$ cells in response to estrogen stimulation and in an epidermal growth factor receptor-dependent manner. ${ }^{76}$

To the best of our knowledge, there are no reports indicating RSPO2 or RSPO4 contribution in the normal mammary gland development. Regarding RSPO3, we have shown that it is expressed in the basal stem cellenriched compartment of adult virgin mouse mammary glands, whereas it is absent from committed mature luminal cells. ${ }^{38}$ Besides, RSPO3 was detected in the mammary stroma and associated with the ECM, where it could exert a significant effect on the epithelial population. ${ }^{38}$ In addition, in silico analysis revealed that the committed alveolar luminal cell population of midpregnant animals expresses lower levels of Rspo3 than alveolar luminal stem/progenitor mammary cells of virgin mice. ${ }^{38}$ In culture, RSPO3 impaired lactogenic differentiation in luminal-like, functionally normal mammary cells, whereas it promoted the acquisition of fibroblastlike, mesenchymal morphology in mammary epithelial cells through canonical WNT pathway activation, increasing the expression levels of mesenchymal markers as fibronectin and vimentin. ${ }^{38}$ These results suggest that RSPO3 could play a relevant role in the normal mammary gland biology, promoting the maintenance of an uncommitted basal-like mammary epithelial phenotype and inducing EMT-like features, which may be the underlying mechanisms for transforming $\mathrm{BC}$ cells that overexpress RSPO3.

\section{2 | LGR4-6 in normal mammary gland development}

LGR4 is expressed in the mammary basal compartment of virgin female mice. Particularly, Wang et al. demonstrated that Lgr4 promoter was active in most TEBs, as well as in mammary duct basal cells of 6-week-old virgin female mice. ${ }^{34}$ They also showed that LGR4 is required for mammary gland development, since $\mathrm{Lgr}^{-/-}$mice displayed delayed ductal development, decreased sidebranching and a severely diminished MaSCs repopulation capacity. ${ }^{34}$ Besides, mammospheres from $\mathrm{Lgr}^{-/-}$cells had decreased activation of the canonical WNT pathway and diminished expression of its target genes as Sox2, which represents one of the key transcription factors in cell reprogramming. ${ }^{34}$ Interestingly, overexpression of this protein restored mammary regenerative potential of $\mathrm{Lgr}^{-/-}$MaSCs. These authors also observed that organoid branching from those cells was impaired. Nevertheless, WNT3a, but not RSPO1, treatment rescued MaSCs from this deficit. ${ }^{34}$ In the human breast, LGR4 expression was observed in mammary ducts, but not in myoepithelial cells, suggesting that LGR4 might be involved in human mammary development. ${ }^{77}$

LGR5 expression pattern varies during mouse mammary gland development, since it appears confined to the luminal layer until day 12 after birth, but afterward its expression switches to the basal compartment. ${ }^{78}$ Furthermore, it was shown that basal LGR5 ${ }^{+}$cells are able to efficiently reconstitute mammary glands in vivo upon serial transplantations. ${ }^{33}$ Using a three-dimensional (3D) imaging strategy and a stochastic multicolor Cre reporter, it has been observed that both luminal and basal mammary cell patches exhibited LGR5 expression along the ductal tree and contributed to alveoli formation, establishing the bipotential capacity of LGR5 ${ }^{+}$mammary cells. ${ }^{79}$ Nevertheless, it has to be noticed that transgenic and knock-in Cre drivers used to perform lineage-tracing experiments are commonly heterogeneously expressed, compromising the interpretation of the obtained data. In fact, more recently, a rigorous assessment of these studies has shown that only unipotent progenitors would be involved in postnatal development and adult tissue remodeling of the mouse mammary gland. ${ }^{80}$ 
Interestingly, Zhang et al. found that mammary organoids derived from murine $\mathrm{LGR}^{+}$single cells were sustainable during prolonged passaging using a growth factor cocktail containing EGF, WNT3a, and RSPO1, and consisted of at least four distinct cell populations self-organized into ductal-like structures. ${ }^{81}$ Through gene expression profiling of mouse LGR $^{+}$basal epithelial cells, a new marker of MaSC subpopulations, Tetraspanin8 (Tspan8), has been proposed. ${ }^{82}$ Interestingly, LGR $5^{+}$Tspan $8{ }^{\text {hi }}$ mouse mammary cell subset showed a remarkably similar expression profile to human Claudin-low tumors. ${ }^{82}$ Nevertheless, it has been indicated that LGR5 expression would not be, by itself, a reliable marker of the mammary progenitor population since protein $\mathrm{C}$ receptor (PROCR) expressing cells, even without detectable LGR5 expression (LGR5 ${ }^{-} \mathrm{PROCR}^{+}$ mammary cells) showed regenerative capacity, whereas $\mathrm{LGR}^{+} \mathrm{PROCR}^{-}$cells were unable to form colonies in vitro and had lower repopulating frequency than the average basal cells. ${ }^{83}$

LGR6 has been reported as a relevant marker of unipotent progenitor cells in the luminal and basal compartments of the mouse mammary gland. ${ }^{84}$ Lineagetracing analysis showed that LGR6+ cells expand clonally during puberty and, with the onset of pregnancy or upon hormone stimulation, they regain proliferative potency contributing to the development of functional alveoli. $^{84}$

Taking together, these studies show a presumably distinct expression pattern and role of each LGR during different stages throughout the mouse mammary gland development. However, whether these reported differences depend on the availability of specific locally secreted RSPOs, or even a potentially overlapping function among LGR4, LGR5, and LGR6 in maintaining the mammary gland homeostasis, remain to be elucidated. Moreover, the precise upstream mechanism regulating LGR4-6 expression in this tissue is unexplored.

\section{3 | RSPO/LGR signaling potentiation in mammary tumor initiation and maintenance}

\subsection{1 | RSPOs in mouse mammary tumors and human $B C$}

Rspo2 and Rspo3 sequences were first identified because of their proximity to common MMTV integration sites in mammary tumors induced by this retrovirus in inbred mice. ${ }^{85-87}$ In addition, overexpression of these genes was involved in mammary tumor initiation and progression..$^{85-87}$ More recently, we have shown that RSPO3 induces mesenchymallike features and migration capacity in nontumorigenic mouse mammary cells. ${ }^{38}$ Besides, we found that canonical WNT pathway activation and in vivo tumorigenicity of basal mouse mammary cancer cells depend on RSPO3 endogenous expression. ${ }^{38}$ Regarding the regulation of RSPO3 expression, it was reported that the runt-related transcription factor 1 (RUNX1) can bind to the Rspo3 promoter region, upregulating its levels in mammary tumor cells. ${ }^{88}$ In the case of RSPO1 induction in BC, a synergy between MYC and the long noncoding RNA PVT1, has been proposed. ${ }^{89}$ In addition, Yoldi et al..$^{90}$ reported that RANK signaling induces RSPO1 expression in MMTV-PyMT tumor cells, as previously reported in the normal mouse mammary gland. ${ }^{75}$

In human $\mathrm{BC}$, it has been reported that $\mathrm{RSPO} 2$ as well as $\mathrm{RSPO} 4$ are overexpressed in triple-negative and metaplastic tumors. ${ }^{91}$ In addition, even though RSPO3 expression was detected in both Luminal and Basal BC, the highest levels of expression also corresponded to the last subtype. ${ }^{38}$ However, RSPO3 overexpression was even more pronounced in Claudin-low BC compared with any other category, if this subtype is considered separately. ${ }^{38}$ Here, an updated analysis of the The Cancer Genome Atlas (TCGA)-BC RNA-Seq dataset composed by 1,097 primary breast carcinomas samples obtained from the UCSC Xena Browser (https://xenabrowser.net), performed as described in Tocci et al., ${ }^{38}$ is displayed (Figure 3a). This approach rendered similar results to what previously reported, ${ }^{38,91}$ indicating that RSPO3 and RSPO4 expression prevail in Basal-like tumors, while RSPO1 is particularly overexpressed in the Luminal A subtype. It would be interesting to determine whether RSPO1 overrepresentation in $\mathrm{ER}^{+} \mathrm{PR}^{+} \mathrm{BC}$ might be related to the previously reported indirect-dependence of this gene expression on progesterone circulating levels. ${ }^{74}$ Unfortunately, we were not able to include RSPO2 in Figure $3 \mathrm{a}$ because its expression levels in the analyzed samples were too low to draw any conclusion. As an overview, Figure 4 shows a graphical abstract depicting the expression pattern and activity of RSPO1 and RSPO3 in the normal adult mammary gland and among $\mathrm{BC}$ subtypes.

\subsection{2 | LGR4-6 and ZNRF3/RNF43 in mammary tumors}

Differently from the well-documented role of LGRs in MaSCs activity and normal mammary gland development, little is known about their role in BCSCs, BC subtypes, and their progression. However, it has been reported that LGR4-6 is overexpressed in the Basal-like subtype and that LGR levels correlate with RSPO, EMT, and stem cell marker expression in human BC. ${ }^{38,91}$ In addition, LGR4 down-regulation reduced cell migration, 


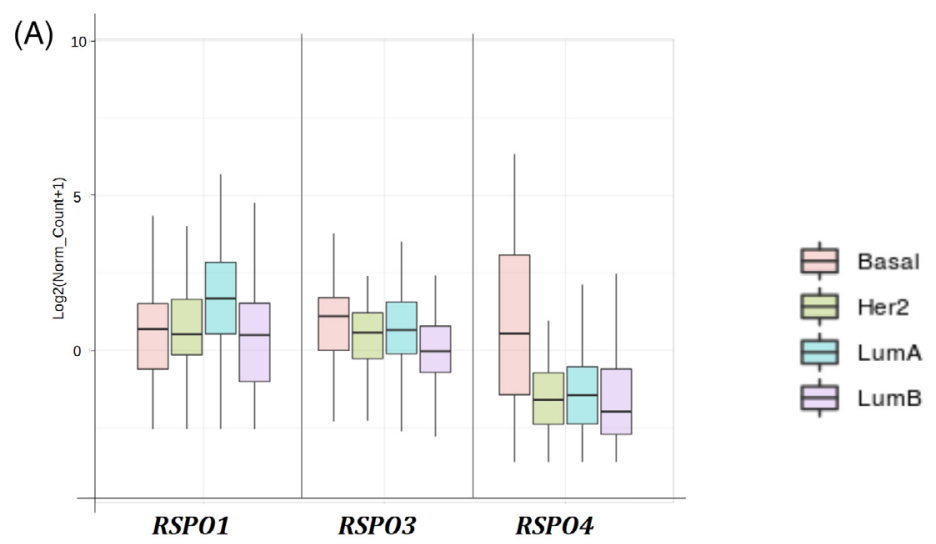

(B)

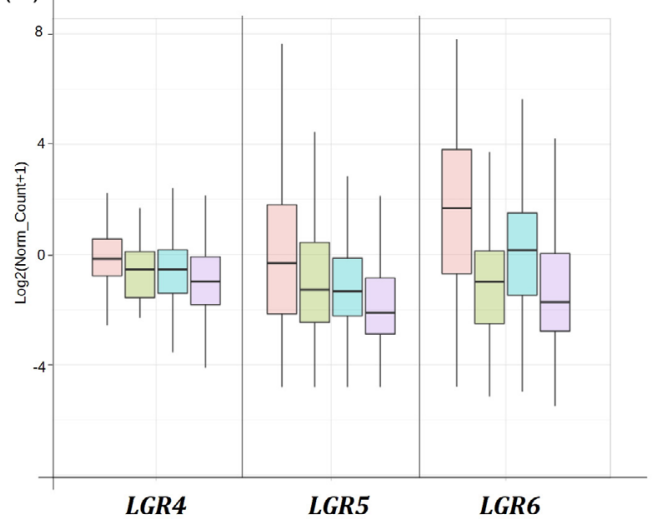

(C)

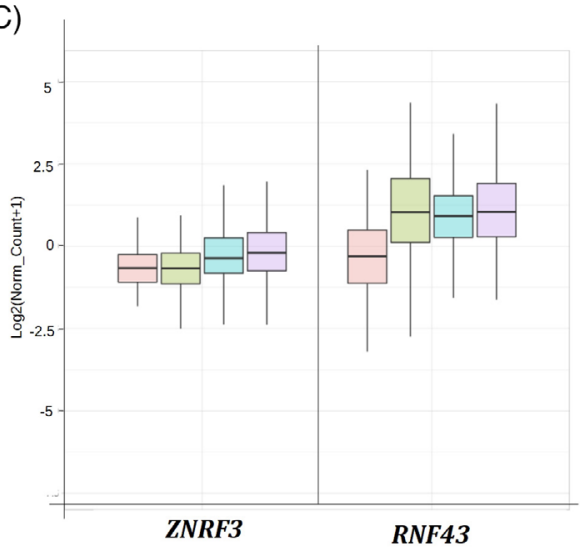

F I G U RE 3 RSPOs, LGRs, and ZNRF3/RNF43 expression levels obtained from a TCGA BC RNA-Seq dataset (1,097 primary breast carcinomas samples) from the UCSC Xena Browser (https://xenabrowser.net). LGR, leucine-rich repeat-containing G-protein-coupled receptor; RSPO, R-spondin; ZNRF3/ RNF43, zinc and RING finger 3/RING finger 43

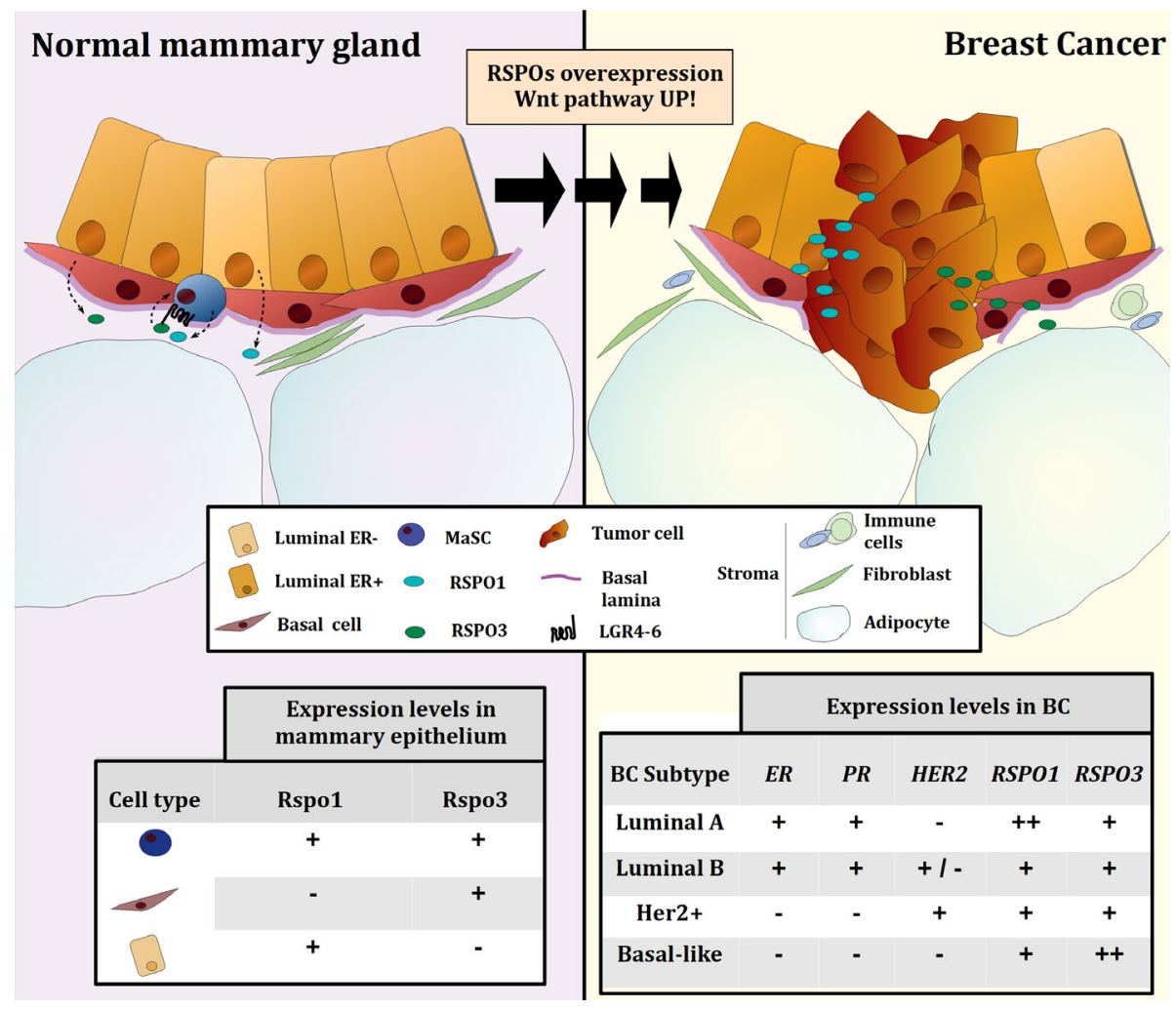

FI G U R E 4 RSPO1 and RSPO3 expression patterns in the normal and neoplastic mammary gland. In the mature normal mammary gland, RSPO1 and $\mathrm{RSPO} 3$ participate in the maintenance of MaSCs. The committed luminal ER-cells and the basal compartment express RSPO1 and RSPO3, respectively. Overexpression of RSPOs and potentiation of the WNT pathway is observed in breast tumors. RSPO1 expression is higher in the Luminal A subtype, while RSPO3 predominates in the Basal BC subtype. $\mathrm{BC}$, breast cancer; ER, estrogen receptor; MaSC, Mammary stem cell; RSPO, Rspondin 
tumor xenograft, and lung metastasis growth of human BC cells and impaired $M M T V$-WNT1 CSC survival upon transplantation in mice. ${ }^{92}$ Regarding LGR5, it has been reported that it promotes $\mathrm{BC}$ progression and the maintenance of stem-like cells through canonical WNT pathway activation. ${ }^{93}$ Besides, analysis of women BC samples revealed LGR5 expression associated with triple-negative BCs, tumor size, and metastasis, as well as with lower relapse-free survival rates. ${ }^{94}$ Finally, patients with high LGR6 expression showed a significantly lower diseasefree survival and it has been demonstrated that $\operatorname{Lgr}^{+}$are potent luminal tumor initiating cells involved in mouse mammary cancer maintenance and growth. ${ }^{84}$ However, this gene is mainly overrepresented in the Basal-like subtype of human BC (Figure 3b, Reference 38). Further studies to obtain a complete understanding on how LGR expression is regulated and how these proteins exert their biological effects under homeostatic conditions and in BC cells remain to be performed. Moreover, dissecting unique or redundant activities of each receptor on cells co-expressing LGRs will be of major relevance for the potential development of $\mathrm{BC}$ therapeutic approaches.

Although RSPOs are LGR ligands, it has been reported-and already mentioned herein-that RSPO2 and RSPO3 are capable of potentiating WNT signaling by binding to the membrane ubiquitin ligases ZNRF3/ RNF43, without interacting with LGRs. ${ }^{28-30}$ Interestingly, our current data-mining analysis reveals that RNF43 is overexpressed in all but the Basal subtype (Figure 3C). Therefore, it could be proposed that RSPOs may play a relevant role in promoting the development of those $\mathrm{BC}$ subtypes showing low LGR levels by directly binding to the RNF43 ubiquitin-ligase protein.

\section{5 | RSPOS AS MOLECULAR TARGETS FOR BC TREATMENT: NEW PERSPECTIVES}

The preeminent RSPO expression in BC undoubtedly supports their putative relevance as therapeutic targets for this cancer type. It has been reported that specific monoclonal antibodies against RSPOs significantly reduced tumor growth of different human cancer types in immuno-deficient mice. ${ }^{36}$ Then, OncoMed Pharmaceuticals produced an anti-RSPO3 blocking monoclonal antibody, named Rosmantuzumab (OMP-131R10), which proved to exert antineoplastic effects on tumor cells in culture and in xenotransplanted mice. ${ }^{95}$ However, a Phase $1 \mathrm{a} / \mathrm{b}$ clinical trial for treatment of solid tumors using Rosmantuzumab as adjuvant therapy did not supply compelling proof of clinical benefit for patients receiving humanized anti-RSPO3 (ClinicalTrials.gov
Identifier: NCT02482441). As described by the authors, during antibody generation and assessment, they only tested the ability of anti-RSPO antibodies to block RSPO interaction with LGRs. ${ }^{36}$ Therefore, considering the fact that the RSPOs are able to interact with other membrane and extracellular components, as ZNRF3/RNF43 and HSPGs, to modulate WNT signaling activation in an LGR-independent manner, a polyvalent therapeutic strategy might be more efficient in reducing RSPO enhancing activity in cancer patients. However, it is conceivable that the efficacy of these putative polyvalent therapies would also depend on the repertoire of WNT ligands, membrane proteins, and mediators of the canonical and noncanonical WNT pathways expressed by tumor cells in each specific BC subtype.

\section{CONCLUDING REMARKS}

WNT pathways play a critical role during mammary gland development through the maintenance and differentiation of MaSCs, and its deregulation has been implicated in BC progression. RSPOs strongly enhance target cell sensitivity to WNT ligands in several tissues through their interaction with LGRs and the E3 ubiquitin-ligases at the cell membrane. RSPO and LGR expressions have been identified during normal mammary gland development, suggesting that this RSPO-LGR axis might play a significant role as a WNT-enhancing signaling cascade in this secretory organ. Under pathological conditions, elevated RSPO and LGR levels have been detected in several cancer models, including $\mathrm{BC}$, indicating that these proteins may be functionally relevant for breast tumorigenesis. However, more studies regarding their precise mechanisms of action, in the diverse scenarios they have been found, are required to determine the proper approach to tackle their impact on $\mathrm{BC}$ progression.

Traditional therapies against cancer, chemo and radiotherapy, have several limitations that lead to treatment failure and cancer recurrence. These limitations are due to systemic and local toxicity, since the agents are not selective enough, and to drug resistance, a property of the CSC population. Thus, eradicating CSCs, by targeting specific cell surface markers and modulating signaling pathways involved in CSC maintenance, becomes essential for cancer treatment by inducing a long-lasting clinical response to prevent tumor relapse. Since the available data indicate that RSPOs and LGRs are relevant for both CSCs and normal adult stem cells of different organs such as the mammary gland, they might be proposed as CSCs markers and molecular targets in cancer therapies. Particularly, for BC patients, therapeutic strategies that may alter the normal MaSC 
compartment would not represent a clinical disadvantage, due to the nonvital character of this organ. However, since RSPO-LGR axis plays a relevant role in regulating the WNT pathway, critically required in tissue homeostasis, anti-RSPOs and anti-LGRs therapeutic strategies should be pursued hand in hand with specific delivery systems to avoid stem cell depletion in essential organs. Taking into account that a main goal of $\mathrm{BC}$ treatment is to prevent the development of distant metastasis, which may require systemic approaches, determining specific features of the RSPO-LGR axis in BC cells is crucial to generate valid therapeutic strategies.

\section{ACKNOWLEDGMENTS}

The authors thank Omar A. Coso for critical reading of the manuscript. This study was supported by CONICET, University of Buenos Aires (UBACYT 20020160100087BA), and by the ANPCyT (PICT 2016-2834), Argentina.

\section{REFERENCES}

1. Clevers H. WNT/beta-catenin signaling in development and disease. Cell. 2006;127:469-480.

2. Anastas JN, Moon RT. WNT signalling pathways as therapeutic targets in cancer. Nat Rev Cancer. 2013;13:11-26.

3. Willert K, Brown JD, Danenberg E, et al. WNT proteins are lipid-modified and can act as stem cell growth factors. Nature. 2003;423:448-452.

4. Kiecker C, Niehrs C. A morphogen gradient of WNT/betacatenin signalling regulates anteroposterior neural patterning in Xenopus. Development. 2001;128:4189-4201.

5. Gross JC, Chaudhary V, Bartscherer K, Boutros M. Active WNT proteins are secreted on exosomes. Nat Cell Biol. 2012; 14:1036-1045.

6. Stanganello E, Hagemann AI, Mattes B, Sinner C, Meyen D, et al. Filopodia-based WNT transport during vertebrate tissue patterning. Nat Commun. 2015;6:5846.

7. Farin HF, Jordens I, Mosa MH, et al. Visualization of a shortrange WNT gradient in the intestinal stem-cell niche. Nature. 2016;530:340-343.

8. Port F, Basler K. WNT trafficking: New insights into WNT maturation, secretion and spreading. Traffic. 2010;11:1265-1271.

9. Routledge D, Scholpp S. Mechanisms of intercellular WNT transport. Development. 2019;146:dev176073.

10. Niehrs C. The complex world of WNT receptor signalling. Nat Rev Mol Cell Biol. 2012;13:767-779.

11. Gómez-Orte E, Sáenz-Narciso B, Moreno S, Cabello J. Multiple functions of the noncanonical WNT pathway. Trends Genet. 2013;29:545-553.

12. Kazanskaya O, Glinka A, del Barco Barrantes I, Stannek P, Niehrs C, Wu W. R-Spondin2 is a secreted activator of WNT/beta-catenin signaling and is required for Xenopus myogenesis. Dev Cell. 2004;7:525-534.

13. de Lau WB, Snel B, Clevers HC. The R-spondin protein family. Genome Biol. 2012;13:242.

14. Yoon JK, Lee JS. Cellular signaling and biological functions of R-spondins. Cell Signal. 2012;24:369-377.
15. Carmon KS, Gong X, Lin Q, Thomas A, Liu Q. R-spondins function as ligands of the orphan receptors LGR4 and LGR5 to regulate WNT/ $\beta$-catenin signaling. Proc Natl Acad Sci U S A. 2011;108:11452-11457.

16. Zebisch $\mathrm{M}, \mathrm{Xu} \mathrm{Y}, \mathrm{Krastev} \mathrm{C}$, et al. Structural and molecular basis of ZNRF3/RNF43 transmembrane ubiquitin ligase inhibition by the WNT agonist R-spondin. Nat Commun. 2013;4: 2787.

17. Nam JS, Turcotte TJ, Smith PF, Choi S, Yoon JK. Mouse cristin/R-spondin family proteins are novel ligands for the frizzled 8 and LRP6 receptors and activate beta-catenin-dependent gene expression. J Biol Chem. 2006;281:13247-13257.

18. Ohkawara B, Glinka A, Niehrs C. Rspo3 binds syndecan 4 and induces WNT/PCP signaling via clathrin-mediated endocytosis to promote morphogenesis. Dev Cell. 2011;20:303-314.

19. Ren Z, van Andel H, de Lau W, et al. Syndecan-1 promotes WNT/beta-catenin signaling in multiple myeloma by presenting WNTs and R-spondins. Blood. 2018;131:982-994.

20. Barker N, Tan S, Clevers H. Lgr proteins in epithelial stem cell biology. Development. 2013;140:2484-2494.

21. Chen PH, Chen X, Lin Z, Fang D, He X. The structural basis of R-spondin recognition by LGR5 and RNF43. Genes Dev. 2013; 27:1345-1350.

22. Hao HX, Xie Y, Zhang Y, et al. ZNRF3 promotes WNT receptor turnover in an R-spondin-sensitive manner. Nature. 2012;485: 195-200.

23. Koo B-K, Spit M, Jordens I, et al. Tumour suppressor RNF43 is a stem-cell E3 ligase that induces endocytosis of WNT receptors. Nature. 2012;488:665-669.

24. de Lau W, Peng WC, Gros P, Clevers H. The Rspondin/Lgr5/Rnf43 module: Regulator of WNT signal strength. Genes Dev. 2014;28:305-316.

25. de Lau W, Barker N, Low TY, Koo BK, Li VS, et al. Lgr5 homologues associate with WNT receptors and mediate R-spondin signalling. Nature. 2011;476:293-297.

26. Carmon KS, Lin Q, Gong X, Thomas A, Liu Q. Correction for Carmon et al., "LGR5 interacts and cointernalizes with WNT receptors to modulate WNT/ $\beta$-catenin signaling”. Mol Cell Biol. 2017;37:e00203-e00217.

27. Carmon KS, Gong X, Yi J, Thomas A, Liu Q. RSPO-LGR4 functions via IQGAP1 to potentiate WNT signaling. Proc Natl Acad Sci U S A. 2014;111:E1221-E1229.

28. Lebensohn AM, Rohatgi R. R-spondins can potentiate WNT signaling without LGRs. Elife. 2018;7:e33126.

29. Park S, Cui J, Yu W, Wu L, Carmon KS, Liu QJ. Differential activities and mechanisms of the four R-spondins in potentiating WNT/beta-catenin signaling. J Biol Chem. 2018;293: 9759-9769.

30. Szenker-Ravi E, Altunoglu U, Leushacke M, Bosso-Lefevre C, Khatoo M, et al. RSPO2 inhibition of RNF43 and ZNRF3 governs limb development independently of LGR4/5/6. Nature. 2018;557:564-569.

31. Park PW, Reizes O, Bernfield M. Cell surface heparan sulfate proteoglycans: Selective regulators of ligand-receptor encounters. J Biol Chem. 2000;275:29923-29926.

32. Jin YR, Yoon JK. The R-spondin family of proteins: Emerging regulators of WNT signaling. Int J Biochem Cell Biol. 2012;44: $2278-2287$ 
33. Plaks V, Brenot A, Lawson DA, Linnemann JR, Van Kappel EC, et al. Lgr5-expressing cells are sufficient and necessary for postnatal mammary gland organogenesis. Cell Rep. 2013;3:70-78.

34. Wang Y, Dong J, Li D, et al. Lgr4 regulates mammary gland development and stem cell activity through the pluripotency transcription factor Sox2. Stem Cells. 2013;31:1921-1931.

35. Seshagiri S, Stawiski EW, Durinck S, et al. Recurrent Rspondin fusions in colon cancer. Nature. 2012;488:660-664.

36. Chartier C, Raval J, Axelrod F, et al. Therapeutic targeting of tumor-derived R-Spondin attenuates beta-catenin signaling and tumorigenesis in multiple cancer types. Cancer Res. 2016; 76:713-723.

37. Gong X, Yi J, Carmon KS, et al. Aberrant RSPO3-LGR4 signaling in Keap1-deficient lung adenocarcinomas promotes tumor aggressiveness. Oncogene. 2015;34:4692-4701.

38. Tocci JM, Felcher CM, Garcia Sola ME, Goddio MV, Zimberlin $\mathrm{MN}$, et al. R-spondin3 is associated with basalprogenitor behavior in normal and tumor mammary cells. Cancer Res. 2018;78:4497-4511.

39. Blanpain C, Fuchs E. Stem cell plasticity. of epithelial stem cells in tissue regeneration. Science. 2014;344:1242281.

40. Hens JR, Wysolmerski JJ. Key stages of mammary gland development: Molecular mechanisms involved in the formation of the embryonic mammary gland. Breast Cancer Res. 2005;7:220-224.

41. McNally S, Stein T. Overview of mammary gland development: A comparison of mouse and human. Methods Mol Biol. 2017; 1501:1-17.

42. Russo J, Russo IH. Development of the human breast. Maturitas. 2004;49:2-15.

43. Richert MM, Schwertfeger KL, Ryder JW, Anderson SM. An atlas of mouse mammary gland development. J Mammary Gland Biol Neoplasia. 2000;5:227-241.

44. Kordon EC, Smith GH. An entire functional mammary gland may comprise the progeny from a single cell. Development. 1998;125:1921-1930.

45. Shackleton M, Vaillant F, Simpson KJ, et al. Generation of a functional mammary gland from a single stem cell. Nature. 2006;439:84-88.

46. Oakes SR, Gallego-Ortega D, Ormandy CJ. The mammary cellular hierarchy and breast cancer. Cell Mol Life Sci. 2014;71: 4301-4324.

47. Al-Hajj M, Wicha MS, Benito-Hernandez A, Morrison SJ, Clarke MF. Prospective identification of tumorigenic breast cancer cells. Proc Natl Acad Sci U S A. 2003;100:3983-3988.

48. Prat A, Pineda E, Adamo B, Galvan P, Fernandez A, et al. Clinical implications of the intrinsic molecular subtypes of breast cancer. Breast. 2015;24(Suppl 2):S26-S35.

49. Nishimura R, Arima N. Is triple negative a prognostic factor in breast cancer? Breast Cancer. 2008;15:303-308.

50. Prat A, Parker JS, Karginova O, et al. Phenotypic and molecular characterization of the claudin-low intrinsic subtype of breast cancer. Breast Cancer Res. 2010;12:R68.

51. Incassati A, Chandramouli A, Eelkema R, Cowin P. Key signaling nodes in mammary gland development and cancer: $\beta$-catenin. Breast Cancer Res. 2010;12:213-213.

52. Roarty K, Rosen JM. WNT and mammary stem cells: Hormones can't fly wingless. Curr Opin Pharmacol. 2010;10:643-649.

53. Chu EY, Hens J, Andl T, et al. Canonical WNT signaling promotes mammary placode development and is essential for initiation of mammary gland morphogenesis. Development. 2004;131:4819-4829.

54. Liu BY, McDermott SP, Khwaja SS, Alexander CM. The transforming activity of WNT effectors correlates with their ability to induce the accumulation of mammary progenitor cells. Proc Natl Acad Sci U S A. 2004;101:4158-4163.

55. Lindvall C, Zylstra CR, Evans N, et al. The WNT co-receptor Lrp6 is required for normal mouse mammary gland development. PLoS One. 2009;4:e5813.

56. van Amerongen R, Bowman AN, Nusse R. Developmental stage and time dictate the fate of WNT/beta-catenin-responsive stem cells in the mammary gland. Cell Stem Cell. 2012;11: 387-400.

57. Teissedre B, Pinderhughes A, Incassati A, Hatsell SJ, Hiremath M, Cowin P. MMTV-WNT1 and $-\Delta$ N89 $\beta$-catenin induce canonical signaling in distinct progenitors and differentially activate hedgehog signaling within mammary tumors. PLoS One. 2009;4:e4537. https://doi.org/10.1371/annotation/ 3706d1475-e1082-1374be1376-b1328-1377d1378aea1302b1986

58. Nakopoulou L, Mylona E, Papadaki I, et al. Study of phosphobeta-catenin subcellular distribution in invasive breast carcinomas in relation to their phenotype and the clinical outcome. Mod Pathol. 2006;19:556-563.

59. Khramtsov AI, Khramtsova GF, Tretiakova M, Huo D, Olopade OI, Goss KH. WNT/beta-catenin pathway activation is enriched in basal-like breast cancers and predicts poor outcome. Am J Pathol. 2010;176:2911-2920.

60. Geyer FC, Lacroix-Triki M, Savage K, et al. Beta-catenin pathway activation in breast cancer is associated with triplenegative phenotype but not with CTNNB1 mutation. Mod Pathol. 2011;24:209-231.

61. Roarty K, Pfefferle AD, Creighton CJ, Perou CM, Rosen JM. Ror2-mediated alternative WNT signaling regulates cell fate and adhesion during mammary tumor progression. Oncogene. 2017;36:5958-5968.

62. Xu J, Prosperi JR, Choudhury N, Olopade OI, Goss KH. Betacatenin is required for the tumorigenic behavior of triplenegative breast cancer cells. PLoS One. 2015;10:e0117097.

63. Ahmed K, Koval A, Xu J, Bodmer A, Katanaev VL. Towards the first targeted therapy for triple-negative breast cancer: Repositioning of clofazimine as a chemotherapy-compatible selective WNT pathway inhibitor. Cancer Lett. 2019;449:45-55.

64. Ji H, Goode RJ, Vaillant F, Mathivanan S, Kapp EA, et al. Proteomic profiling of secretome and adherent plasma membranes from distinct mammary epithelial cell subpopulations. Proteomics. 2011;11:4029-4039.

65. Roarty K, Serra R. WNT5a is required for proper mammary gland development and TGF-beta-mediated inhibition of ductal growth. Development. 2007;134:3929-3939.

66. Kessenbrock K, Smith P, Steenbeek SC, et al. Diverse regulation of mammary epithelial growth and branching morphogenesis through noncanonical WNT signaling. Proc Natl Acad Sci U S A. 2017;114:3121-3126.

67. Kessenbrock K, Dijkgraaf GJ, Lawson DA, Littlepage LE, Shahi $\mathrm{P}$, et al. A role for matrix metalloproteinases in regulating mammary stem cell function via the WNT signaling pathway. Cell Stem Cell. 2013;13:300-313.

68. Klemm F, Bleckmann A, Siam L, Chuang HN, Rietkotter E, et al. Beta-catenin-independent WNT signaling in basal-like 
breast cancer and brain metastasis. Carcinogenesis. 2011;32: 434-442.

69. Shi J, Wang Y, Zeng L, et al. Disrupting the interaction of BRD4 with diacetylated twist suppresses tumorigenesis in basal-like breast cancer. Cancer Cell. 2014;25:210-225.

70. Cao J, Wang X, Dai T, et al. Twist promotes tumor metastasis in basal-like breast cancer by transcriptionally upregulating ROR1. Theranostics. 2018;8:2739-2751.

71. Tomizuka K, Horikoshi K, Kitada R, et al. R-spondin1 plays an essential role in ovarian development through positively regulating WNT-4 signaling. Hum Mol Genet. 2008;17:1278-1291.

72. Chadi S, Buscara L, Pechoux C, et al. R-spondin1 is required for normal epithelial morphogenesis during mammary gland development. Biochem Biophys Res Commun. 2009;390:1040-1043.

73. Chadi S, Polyte J, Lefevre L, et al. Phenotypic and molecular alterations in the mammary tissue of R-Spondin1 Knock-out mice during pregnancy. PLoS One. 2016;11:e0162566.

74. Cai C, Yu QC, Jiang W, et al. R-spondin1 is a novel hormone mediator for mammary stem cell self-renewal. Genes Dev. 2014;28:2205-2218.

75. Joshi PA, Waterhouse PD, Kannan N, et al. RANK signaling amplifies WNT-responsive mammary progenitors through RSPONDIN1. Stem Cell Rep. 2015;5:31-44.

76. Cai C, Geng A, Wang M, Yang L, Yu QC, et al. Amphiregulin mediates the hormonal regulation on Rspondin-1 expression in the mammary gland. Dev Biol. 2020;458:43-51.

77. Yi J, Xiong W, Gong X, Bellister S, Ellis LM, Liu Q. Analysis of LGR4 receptor distribution in human and mouse tissues. PLoS One. 2013;8:e78144.

78. de Visser KE, Ciampricotti M, Michalak EM, Tan DW, Speksnijder EN, et al. Developmental stage-specific contribution of LGR5(+) cells to basal and luminal epithelial lineages in the postnatal mammary gland. J Pathol. 2012;228:300-309.

79. Rios AC, Fu NY, Lindeman GJ, Visvader JE. In situ identification of bipotent stem cells in the mammary gland. Nature. 2014;506:322-327.

80. Wuidart A, Ousset M, Rulands S, Simons BD, Van Keymeulen A, et al. Quantitative lineage tracing strategies to resolve multipotency in tissue-specific stem cells. Genes Dev. 2016;30:1261-1277.

81. Zhang L, Adileh M, Martin ML, et al. Establishing estrogenresponsive mouse mammary organoids from single Lgr5(+) cells. Cell Signal. 2017;29:41-51.

82. Fu NY, Rios AC, Pal B, et al. Identification of quiescent and spatially restricted mammary stem cells that are hormone responsive. Nat Cell Biol. 2017;19:164-176.

83. Wang D, Cai C, Dong X, et al. Identification of multipotent mammary stem cells by protein $\mathrm{C}$ receptor expression. Nature. 2015;517:81-84.
84. Blaas L, Pucci F, Messal HA, Andersson AB, Josue RE, et al. Lgr6 labels a rare population of mammary gland progenitor cells that are able to originate luminal mammary tumours. Nat Cell Biol. 2016;18:1346-1356.

85. Gattelli A, Zimberlin MN, Meiss RP, Castilla LH, Kordon EC. Selection of early-occurring mutations dictates hormoneindependent progression in mouse mammary tumor lines. J Virol. 2006;80:11409-11415.

86. Theodorou V, Kimm MA, Boer M, et al. MMTV insertional mutagenesis identifies genes, gene families and pathways involved in mammary cancer. Nat Genet. 2007;39:759-769.

87. Klauzinska M, Baljinnyam B, Raafat A, et al. Rspo2/Int7 regulates invasiveness and tumorigenic properties of mammary epithelial cells. J Cell Physiol. 2012;227:1960-1971.

88. Recouvreux MS, Grasso EN, Echeverria PC, Rocha-Viegas L, Castilla LH, et al. RUNX1 and FOXP3 interplay regulates expression of breast cancer related genes. Oncotarget. 2016;7: 6552-6565.

89. Sarver AL, Murray CD, Temiz NA, Tseng Y-Y, Bagchi A. MYC and PVT1 synergize to regulate RSPO1 levels in breast cancer. Cell Cycle. 2016;15:881-885.

90. Yoldi G, Pellegrini P, Trinidad EM, et al. RANK signaling blockade reduces breast cancer recurrence by inducing tumor cell differentiation. Cancer Res. 2016;76:5857-5869.

91. Coussy F, Lallemand F, Vacher S, et al. Clinical value of Rspondins in triple-negative and metaplastic breast cancers. Br J Cancer. 2017;116:1595-1603.

92. Yue Z, Yuan Z, Zeng L, et al. LGR4 modulates breast cancer initiation, metastasis, and cancer stem cells. FASEB J. 2018;32: 2422-2437.

93. Yang L, Tang H, Kong Y, et al. LGR5 promotes breast cancer progression and maintains stem-like cells through activation of WNT/ $\beta$-catenin signaling. Stem Cells. 2015;33:2913-2924.

94. Hou MF, Chen PM, Chu PY. LGR5 overexpression confers poor relapse-free survival in breast cancer patients. BMC Cancer. 2018;18:219.

95. Fischer MM, Yeung VP, Cattaruzza F, et al. RSPO3 antagonism inhibits growth and tumorigenicity in colorectal tumors harboring common WNT pathway mutations. Sci Rep. 2017;7:15270.

How to cite this article: Tocci JM, Felcher CM, García Solá ME, Kordon EC. R-spondin-mediated WNT signaling potentiation in mammary and breast cancer development. IUBMB Life. 2020;1-14. https://doi.org/10.1002/iub.2278 